\title{
On L-fuzzy closure operators and L-fuzzy pre-proximities
}

\author{
A. A. Ramadan*, E. H. Elkordy and M. A. Usama
}

*Correspondence:

ahmed.ramadan@science.

bsu.edu.eg

Mathematics and Computer

Science Department,

Faculty of Science, Beni-Suef

University, Beni Suef, Egypt

\begin{abstract}
The aim of this paper is to investigate the relations among the L-fuzzy pre-proximities, $L$-fuzzy closure operators and L-fuzzy co-topologies in complete residuated lattices. We show that there is a Galois correspondence between the category of separated L-fuzzy closure spaces and that of separated L-fuzzy pre-proximity spaces and we give their examples.
\end{abstract}

Keywords: Complete residuated lattice, L-fuzzy pre-proximity, L-fuzzy closure operators, L-fuzzy co-topologies, Galois correspondence

Mathematics Subject Classification: 54F05, 54D10, 54B05, 54B10

\section{Introduction}

Closure operators are very useful tool in several areas of mathematical structures with direct applications, both mathematical (e.g, topology, logic) and extra-mathematical (e.g, data mining, knowledge representation). In fuzzy set theory [1, 2], several particular kinds such as general theory of closure operators which operate with fuzzy sets (socalled fuzzy closure operators) are studied [3-6].

Ward et al. [7] introduced a complete residuated lattice which is an algebraic structure for many valued logic. Bělohlávek [8] investigated information systems, decision rules and developed the notion of fuzzy contexts using Galois connections with $R \in L^{X \times Y}$ on a complete residuated lattices. Höhle [9] introduced $L$-fuzzy topologies with algebraic structure $L$ (cqm, quantales, $M V$-algebra). It has developed in many directions [10-12]. Recently, Bělohlávek [13,14] outlined a general theory of fuzzy closure operators by using the structure of the residuated lattice in place of the usual structure of truth value on $[0,1]$. Fang and Yue [15] studied the relationship between $L$-fuzzy closure systems and $L$-fuzzy topological spaces from a category viewpoint for a complete residuated lattice $L$ (see also [16]). Ramadan [17] studied the relationship between $L$-fuzzy interior systems and $L$-fuzzy topological spaces over complete residuated lattices.

Proximity is an important concept in topology, and it can be considered either as axiomatizations of geometric notions, close to but quite independent of topology, or as convenient tools for an investigation of topological spaces. Hence, proximity has close relations with topology, uniformity and metric. With the development of topology, the

(c) The Author(s) 2021. Open Access This article is licensed under a Creative Commons Attribution 4.0 International License, which permits use, sharing, adaptation, distribution and reproduction in any medium or format, as long as you give appropriate credit to the original author(s) and the source, provide a link to the Creative Commons licence, and indicate if changes were made. The images or other third party material in this article are included in the article's Creative Commons licence, unless indicated otherwise in a credit line to the material. If material is not included in the article's Creative Commons licence and your intended use is not permitted by statutory regulation or exceeds the permitted use, you will need to obtain permission directly from the copyright holder. To view a copy of this licence, visit http:// creativecommons.org/licenses/by/4.0/. 
theory of proximity makes a massive progress. In the framework of $L$-topology, many authors generalized the crisp proximity to $L$-fuzzy setting. Katsaras $[18,19]$ introduced the concepts of fuzzy topogenous order and fuzzy topogenous structures in completely distributive lattice which are a unified approach to the three spaces: Chang's fuzzy topologies [20], Katsaras's fuzzy proximities [21] and Hutton's fuzzy uniformities [22] (see also [23]) . Subsequently, Liu [24], Artico and Moresco [25] extended it into L-fuzzy set theory in view points of Lowen's fuzzy topology [26]. As an extension of Katsaras's definition, El-Dardery [27] introduced $L$-fuzzy topogenous order in view points of Sostak's fuzzy topology [28], smooth fuzzy topology [29] and Kim's $L$-fuzzy proximities [30] on strictly two-sided, commutative quantales. $L$-fuzzy topogenous structures and $L$-fuzzy proximities [23, 31-34] have been developed in a slightly different sense.

In this paper, we introduce the notions of $L$-fuzzy pre-proximities and $L$-fuzzy closure operators in complete residuated lattices. Moreover, we investigate the relations among the $L$-fuzzy pre-proximities, $L$-fuzzy closure operators and $L$-fuzzy co-topologies. We show that there is a Galois correspondence between the category of separated $L$-fuzzy closure spaces and that of separated $L$-fuzzy pre-proximity spaces. In Example 19, as an information system as an extension of Pawlak's rough set [35, 36], $L$-fuzzy pre-proximities, $L$-fuzzy co-topologies and $L$-fuzzy closure operators are introduced. By using these concepts, we can apply them to information systems and decision makings [37].

\section{Preliminaries}

Definition $1([8-11,38])$ An algebra $(L, \wedge, \vee, \odot, \rightarrow, \perp, \top)$ is called a complete residuated lattice if it satisfies the following conditions:

(C1) $(L, \leq, \vee, \wedge, \perp, \top)$ is a complete lattice with the greatest element $T$ and the least element $\perp$;

(C2) $(L, \odot, \top)$ is a commutative monoid;

(C3) $x \odot y \leq z$ iff $x \leq y \rightarrow z$ for $x, y, z \in L$.

In this paper, we assume that $\left(L, \leq, \odot,^{*}\right)$ is a complete residuated lattice with an order reversing involution * which is defined by

$$
x \oplus y=\left(x^{*} \odot y^{*}\right)^{*}, x^{*}=x \rightarrow \perp .
$$

$$
\begin{aligned}
& \text { For } \quad \alpha \in L \text { and } f \in L^{X} \text {, we denote } \quad(\alpha \rightarrow f),(\alpha \odot f), \alpha_{X} \in L^{X} \quad \text { as } \\
& (\alpha \rightarrow f)(x)=\alpha \rightarrow f(x),(\alpha \odot f)(x)=\alpha \odot f(x), \alpha_{X}(x)=\alpha, \text { respectively } \\
& \top_{x}(y)=\left\{\begin{array}{ll}
\top, & \text { if } y=x, \\
\perp, & \text { otherwise },
\end{array} \top_{x}^{*}(y)= \begin{cases}\perp, & \text { if } y=x, \\
\top, & \text { otherwise }\end{cases} \right.
\end{aligned}
$$

Some basic properties of the binary operation $\odot$ and residuated operation $\rightarrow$ are collected in the following lemma, and they can be found in many works, for instance [ $8-11$, 38].

Lemma 2 For each $x, y, z, x_{i}, y_{i}, w \in L$, we have the following properties. 
(1) $\top \rightarrow x=x, \perp \odot x=\perp$,

(2) If $y \leq z$, then $x \odot y \leq x \odot z, x \oplus y \leq x \oplus z, x \rightarrow y \leq x \rightarrow z$ and $z \rightarrow x \leq y \rightarrow x$

(3) $x \leq y$ iff $x \rightarrow y=\top$.

(4) $\left(\bigwedge_{i} y_{i}\right)^{*}=\bigvee_{i} y_{i}^{*},\left(\bigvee_{i} y_{i}\right)^{*}=\bigwedge_{i} y_{i}^{*}$,

(5) $x \rightarrow\left(\bigwedge_{i} y_{i}\right)=\bigwedge_{i}\left(x \rightarrow y_{i}\right)$

(6) $\left(\bigvee_{i} x_{i}\right) \rightarrow y=\bigwedge_{i}\left(x_{i} \rightarrow y\right)$

(7) $x \odot\left(\bigvee_{i} y_{i}\right)=\bigvee_{i}\left(x \odot y_{i}\right)$,

(8) $\left(\bigwedge_{i} x_{i}\right) \oplus y=\bigwedge_{i}\left(x_{i} \oplus y\right)$,

(9) $(x \odot y) \rightarrow z=x \rightarrow(y \rightarrow z)=y \rightarrow(x \rightarrow z)$,

(10) $x \odot y=\left(x \rightarrow y^{*}\right)^{*}, x \oplus y=x^{*} \rightarrow y$ and $x \rightarrow y=y^{*} \rightarrow x^{*}$,

(11) $(x \rightarrow y) \odot(z \rightarrow w) \leq(x \odot z) \rightarrow(y \odot w)$,

(12) $x \rightarrow y \leq(x \odot z) \rightarrow(y \odot z)$ and $(x \rightarrow y) \odot(y \rightarrow z) \leq x \rightarrow z$,

(13) $(x \rightarrow y) \odot(z \rightarrow w) \leq(x \oplus z) \rightarrow(y \oplus w)$,

(14) $x \odot(x \rightarrow y) \leq y$ and $y \leq x \rightarrow(x \odot y)$,

(15) $(x \vee y) \odot(z \vee w) \leq(x \vee z) \vee(y \odot w) \leq(x \oplus z) \vee(y \odot w)$,

(16) $\bigvee_{i \in \Gamma} x_{i} \rightarrow \bigvee_{i \in \Gamma} y_{i} \geq \bigwedge_{i \in \Gamma}\left(x_{i} \rightarrow y_{i}\right), \bigwedge_{i \in \Gamma} x_{i} \rightarrow \bigwedge_{i \in \Gamma} y_{i} \geq \bigwedge_{i \in \Gamma}\left(x_{i} \rightarrow y_{i}\right)$,

(17) $(x \odot y) \odot(z \oplus w) \leq(x \odot z) \oplus(y \odot w)$,

(18) $z \rightarrow x \leq(x \rightarrow y) \rightarrow(z \rightarrow y)$ and $y \rightarrow z \leq(x \rightarrow y) \rightarrow(x \rightarrow z)$.

Definition $3 \quad[14,16,39]$ A map $\mathcal{C}: L^{X} \rightarrow L^{X}$ is called an $L$-fuzzy closure operator on $X$ if $\mathcal{C}$ satisfies the following conditions:

(C1) $\mathcal{C}\left(\perp_{X}\right)=\perp_{X}$,

(C2) $\mathcal{C}(f) \geq f$ for all $f \in L^{X}$,

(C3) If $f \leq g$, then $\mathcal{C}(f) \leq \mathcal{C}(g))$ for all $f, g \in L^{X}$,

(C4) $\mathcal{C}(f \oplus g) \leq \mathcal{C}(f) \oplus \mathcal{C}(g)$.

The pair $(X, \mathcal{C})$ is called $L$-fuzzy closure space. An $L$-fuzzy closure space is called

(T) topological if $\mathcal{C}(\mathcal{C}(f))=\mathcal{C}(f) \forall f \in L^{X}$,

(U) stratified if $\mathcal{C}(\alpha \rightarrow f) \leq \alpha \rightarrow \mathcal{C}(f)$ for all $f \in L^{X}$ and $\alpha \in L$,

(V) co-stratified if $\mathcal{C}(\alpha \odot f) \leq \alpha \odot \mathcal{C}(f)$ for all $f \in L^{X}$ and $\alpha \in L$,

(W) strong if it is both stratified and co-stratified, i.e, $\mathcal{C}(\alpha \odot f)=\alpha \odot \mathcal{C}(f)$ for all $f \in L^{X}$ and $\alpha \in L$,

(X) separated if $\mathcal{C}\left(\top_{x}^{*}\right)=T_{x}^{*}$ for all $x \in X$,

(Y) generalized if $\mathcal{C}(f)(x) \geq \bigvee_{x \in X} f(x)$,

(Z) Alexandrov if $\mathcal{C}\left(\bigvee_{i \in \Gamma} f_{i}\right)=\bigvee_{i \in \Gamma} \mathcal{C}\left(f_{i}\right)$.

Definition 4 Let $\left(X, \mathcal{C}_{X}\right)$ and $\left(Y, \mathcal{C}_{Y}\right)$ be $L$-fuzzy closure spaces and $\varphi:\left(X, \mathcal{C}_{X}\right) \rightarrow\left(Y, \mathcal{C}_{Y}\right)$ be a mapping. Then, $D_{C}(\varphi)$ defined by

$$
D_{C}(\varphi)=\bigwedge_{f \in L^{Y}} \bigwedge_{x \in X}\left(\mathcal{C}_{X}\left(\varphi^{\leftarrow}(f)\right)(x) \rightarrow \varphi^{\leftarrow}\left(\mathcal{C}_{Y}(f)\right)(x)\right)
$$


is called the degree of $L F$-closure for $\varphi$. If $D_{C}(\varphi)=\top$, then $\mathcal{C}_{X}\left(\varphi^{\leftarrow}(f)\right) \leq \phi^{\leftarrow}\left(\mathcal{C}_{Y}(f)\right)$ for each $f \in L^{Y}$, which is exactly the definition of $L F$-closure mappings between $L$-fuzzy closure spaces.

\section{Remark 5}

An L-fuzzy closure space $(X, \mathcal{C})$ is stratified if and only if $\mathcal{C}(\alpha \odot f) \geq \alpha \odot \mathcal{C}(f)$.

Definition $6 \quad[16,17,39]$ A mapping $\mathcal{F}: L^{X} \rightarrow L$ is called $L$-fuzzy co-topology on $X$ if it satisfies the following conditions:

(T1) $\mathcal{F}\left(\perp_{X}\right)=\mathcal{F}\left(\top_{X}\right)=\top$,

(T2) $\mathcal{F}(f \oplus g) \geq \mathcal{F}(f) \odot \mathcal{F}(g)$ forall $f, g \in L^{X}$,

(T3) $\mathcal{F}\left(\bigwedge_{i} f_{i}\right) \geq \bigwedge_{i} \mathcal{F}\left(f_{i}\right)$ forall $\left\{f_{i}\right\}_{i \in \Gamma} \subseteq L^{X}$.

The pair $(X, \mathcal{F})$ is called $L$-fuzzy co-topological space. An $L$-fuzzy co-topological space is said to be
(A) stratified if $\mathcal{F}(\alpha \odot f) \geq \mathcal{F}(f)$,
(B) co-stratified if $\mathcal{F}(\alpha \rightarrow f) \geq \mathcal{F}(f)$,
(C) strong if it is both stratified and co-stratified,
(D) separated if $\mathcal{F}\left(\top_{x}\right)=T$ for all $x \in X$,
(E) Alexandrov if $\mathcal{F}\left(\bigvee_{i} f_{i}\right) \geq \bigwedge_{i} \mathcal{F}\left(f_{i}\right)$ forall $\left\{f_{i}\right\}_{i \in \Gamma} \subseteq L^{X}$.

Definition 7 Let $\left(X, \mathcal{F}_{X}\right)$ and $\left(Y, \mathcal{F}_{Y}\right)$ be $L$-fuzzy co-topological spaces and $\varphi:\left(X, \mathcal{F}_{X}\right) \rightarrow\left(Y, \mathcal{F}_{Y}\right)$ be a mapping. Then, $D_{\mathcal{F}}(\phi)$ defined by

$$
D_{\mathcal{F}}(\varphi)=\bigwedge_{f \in L^{Y}}\left(\mathcal{F}_{Y}(f) \rightarrow \mathcal{F}_{X}\left(\varphi^{\leftarrow}(f)\right)\right)
$$

is called the degree of $L F$-continuous for $\varphi$. If $D_{\mathcal{F}}(\varphi)=\top$, then $\mathcal{F}_{Y}(f) \leq \mathcal{F}_{Y}\left(\varphi^{\leftarrow}(f)\right)$ for each $f \in L^{Y}$, which is exactly the definition of $L F$-continuous mappings between $L$-fuzzy co-topological spaces.

Definition $8 \quad[8,36]$ Let $X$ be a set. A map $R: X \times X \rightarrow L$ is called an $L$-partial order if it satisfies the following conditions

(E1) reflexive if $R(x, x)=\top$ for all $x \in X$,

(E2) transitive if $R(x, y) \odot R(y, z) \leq R(x, z)$ for all $x, y, z \in X$,

(E3) antisymmetric if $R(x, y)=R(y, x)=\top$, then $x=y$. 


\section{The relationships between $L$-fuzzy pre-proximities and topological structures}

Definition 9 A mapping $\delta: L^{X} \times L^{X} \rightarrow L$ is called an $L$-fuzzy pre-proximity on $X$ if it satisfies the following axioms.

(P1) $\delta\left(\top_{X}, \perp_{X}\right)=\delta\left(\perp_{X}, \top_{X}\right)=\perp$,

(P2) $\delta(f, g) \geq \bigvee_{x \in X}(f \odot g)(x)$,

(P3) If $f_{1} \leq f_{2}, h_{1} \leq h_{2}$, then $\delta\left(f_{1}, h_{1}\right) \leq \delta\left(f_{2}, h_{2}\right)$. The pair $(X, \delta)$ is called $L$-fuzzy preproximity space. An $L$-fuzzy pre-proximity is called an $(L, \odot, \oplus)$-fuzzy pre-proximity if

(P4) For every $f_{1}, f_{2}, h_{1}, h_{2} \in L^{X}$ we have

$$
\begin{aligned}
& \delta\left(f_{1} \odot f_{2}, h_{1} \oplus h_{2}\right) \leq \delta\left(f_{1}, h_{1}\right) \oplus \delta\left(f_{2}, h_{2}\right), \\
& \delta\left(f_{1} \oplus f_{2}, h_{1} \odot h_{2}\right) \leq \delta\left(f_{1}, h_{1}\right) \oplus \delta\left(f_{2}, h_{2}\right) .
\end{aligned}
$$

An $L$-fuzzy pre-proximity is called an $L$-fuzzy quasi-proximity on $X$ if it satisfies (P4) and

(Q) $\delta(f, g) \geq \bigwedge_{h}\left\{\delta(f, h) \oplus \delta\left(h^{*}, g\right)\right\}$. An $L$-fuzzy quasi-proximity is called an $L$-fuzzy proximity on $X$ if

(P) $\delta^{s}=\delta$ where $\delta^{s}(f, g)=\delta(g, f)$. An $L$-fuzzy pre-proximity is called

(St) stratified if $\delta(\alpha \odot f, \alpha \rightarrow g) \leq \delta(f, g)$ and $\delta(\alpha \rightarrow f, \alpha \odot g) \leq \delta(f, g)$,

(SE) separated if $\delta\left(\top_{x}, T_{x}^{*}\right)=\delta\left(T_{x}^{*}, \top_{x}\right)=\perp$ for each $x \in X$,

(AL) Alexandrov if $\delta\left(\bigvee_{i \in \Gamma} f_{i}, g\right) \leq \bigvee_{i \in \Gamma} \delta\left(f_{i}, g\right), \delta\left(f, \bigvee_{i \in \Gamma} g_{i}\right) \leq \bigvee_{i \in \Gamma} \delta\left(f, g_{i}\right)$,

(GL) generalized if $\delta(f, g) \leq \bigvee_{x \in X} f(x) \odot \bigvee_{x \in X} g(x)$.

Definition 10 Let $\left(X, \delta_{X}\right)$ and $\left(Y, \delta_{Y}\right)$ be $L$-fuzzy pre-proximity spaces and $\varphi:\left(X, \delta_{X}\right) \rightarrow\left(Y, \delta_{Y}\right)$ be a mapping. Then, $D_{\delta}(\varphi)$ defined by

$$
D_{\delta}(\varphi)=\bigwedge_{f, g \in L^{Y}}\left(\delta_{X}\left(\varphi^{\leftarrow}(f), \varphi^{\leftarrow}(g)\right) \rightarrow \delta_{Y}(f, g)\right)
$$

is called the degree of $L F$-proximity for $\varphi$. If $D_{\delta}(\varphi)=\top$, then $\delta_{X}\left(\varphi^{\leftarrow}(f), \varphi^{\leftarrow}(g)\right) \leq \delta_{Y}(f, g)$ for each $f, g \in L^{Y}$, which is exactly the definition of $L F$-proximity mappings between $L$-fuzzy pre-proximity spaces.

Lemma 11 Let $(X, \delta)$ be an L-fuzzy pre-proximity space. Then,

$\delta(\alpha \odot f, g) \geq \alpha \odot \delta(f, g)$ iff $\delta(\alpha \rightarrow f, g) \leq \alpha \rightarrow \delta(f, g)$.

Proof

(1) Let $\delta(\alpha \odot f, g) \geq \alpha \odot \delta(f, g)$. Then, $\alpha \odot \delta(\alpha \rightarrow f, g) \leq \delta(\alpha \odot(\alpha \rightarrow f), g) \leq \delta(f, g)$. Thus, $\delta(\alpha \rightarrow f, g) \leq \alpha \rightarrow \delta(f, g)$.

Let $\delta(\alpha \rightarrow f, g) \leq \alpha \rightarrow \delta(f, g)$. Then, $\delta(f, g) \leq \delta(\alpha \rightarrow \alpha \odot f, g) \leq \alpha \rightarrow \delta(\alpha \odot f, g)$. Thus, $\alpha \odot \delta(f, g) \leq \delta(\alpha \odot f, g)$. 
From the following theorem, we obtain the $L$-fuzzy closure operator induced by an $L$-fuzzy pre-proximity.

Theorem 12 Let $\delta$ be an L-fuzzy pre-proximity on X. Define $\mathcal{C}_{\delta}: L^{X} \rightarrow L^{X}$ as follows:

$$
\mathcal{C}_{\delta}(f)(x)=\bigwedge_{g \in L^{X}}\left\{g(x) \rightarrow \delta\left(g, g^{*}\right) \mid f \leq g^{*}\right\} .
$$

Then,

(1) $\left(X, \mathcal{C}_{\delta}\right)$ is an L-fuzzy closure space,

(2) If $\delta$ is stratified, then $\mathcal{C}_{\delta}$ is stratified,

(3) If $\delta$ is separated, then $\mathcal{C}_{\delta}$ is separated.

Proof

(1)(C1) Since $\delta\left(T_{X}, \perp_{X}\right)=\perp$,

$$
\begin{aligned}
\mathcal{C}_{\delta}\left(\perp_{X}\right)(x) & =\bigwedge_{g \in L^{X}}\left\{g(x) \rightarrow \delta\left(g, g^{*}\right) \mid \perp_{X} \leq g^{*}\right\} \\
& \leq\left(\top_{X}(x) \rightarrow \delta\left(\top_{X}, \perp_{X}\right)\right)=\perp_{X}(x) .
\end{aligned}
$$

(C2) Since $g \leq f^{*}$, then $g \rightarrow \delta\left(g, g^{*}\right) \geq f^{*} \rightarrow \perp=f$.

(C3) If $f \leq h$, then

$$
\begin{aligned}
\mathcal{C}_{\delta}(h)(x) & =\bigwedge_{g \in L^{X}}\left\{\left(g(x) \rightarrow \delta\left(g, g^{*}\right)\right) \mid h \leq g^{*}\right\} \\
& \geq \bigwedge_{g \in L^{X}}\left\{\left(g(x) \rightarrow \delta\left(g, g^{*}\right)\right) \mid f \leq g^{*}\right\}=\mathcal{C}_{\delta}(f)(x) .
\end{aligned}
$$

(C4) Since

$$
\begin{aligned}
& ((a \rightarrow b) \oplus(c \rightarrow d))^{*}=(a \rightarrow b)^{*} \odot(c \rightarrow d)^{*} \\
& =\left(a \odot b^{*}\right) \odot\left(c \odot d^{*}\right)=(a \odot c) \odot\left(b^{*} \odot d^{*}\right),
\end{aligned}
$$

then we have $(a \rightarrow b) \oplus(c \rightarrow d)=(a \odot c) \rightarrow(b \oplus d)$. From Lemma 2, we obtain

$$
\begin{aligned}
\mathcal{C}_{\delta}(f)(x) \oplus \mathcal{C}_{\delta}(h)(x) & =\bigwedge_{g \in L^{X}}\left\{\left(g(x) \rightarrow \delta\left(g, g^{*}\right)\right) \mid f \leq g^{*}\right\} \\
& \oplus \bigwedge_{k \in L^{X}}\left\{\left(k(x) \rightarrow \delta\left(k, k^{*}\right)\right) \mid h \leq k^{*}\right\} \\
& =\bigwedge_{g, k \in L^{X}}\left\{(g(x) \odot k(x)) \rightarrow\left(\delta\left(g, g^{*}\right) \oplus \delta\left(k, k^{*}\right)\right) \mid f \leq g^{*}, h \leq k^{*}\right\} \\
& \left.\left.\geq \bigwedge_{g, k \in L^{X}}\{(g \odot k)(x)) \rightarrow \delta\left(g \odot k, g^{*} \oplus k^{*}\right)\right) \mid f \oplus h \leq g^{*} \oplus k^{*}\right\} \\
& \geq \mathcal{C}_{\delta}(f \oplus h)(x) .
\end{aligned}
$$

Hence, $\mathcal{C}_{\delta}$ is an $L$-fuzzy closure operator on $X$.

(2)

$$
\begin{aligned}
\alpha \rightarrow \mathcal{C}_{\delta}(f) & =\alpha \rightarrow \bigwedge_{g \in L^{X}}\left\{\left(g(x) \rightarrow \delta\left(g, g^{*}\right)\right) \mid f \leq g^{*}\right\} \\
& =\bigwedge_{g \in L^{X}}\left\{\left((\alpha \odot g(x)) \rightarrow \delta\left(g, g^{*}\right)\right) \mid f \leq g^{*}\right\} \\
& \geq \bigwedge_{g \in L^{X}}\left\{(\alpha \odot g(x)) \rightarrow \delta\left(\left(\alpha \odot g, \alpha \rightarrow g^{*}\right)\right) \mid \alpha \rightarrow f \leq \alpha \rightarrow g^{*}\right\} \\
& \geq \mathcal{C}_{\delta}(\alpha \rightarrow f) .
\end{aligned}
$$

(3) By (C2) and 


$$
\mathcal{C}_{\delta}\left(\top_{x}^{*}\right)(x)=\bigwedge_{g \in L^{X}}\left\{g(x) \rightarrow \delta\left(g, g^{*}\right) \mid \top_{x}^{*} \leq g^{*}\right\} \leq \top_{x}(x) \rightarrow \delta\left(\top_{x}, \top_{x}^{*}\right)=\top_{x}^{*},
$$

we have $\mathcal{C}_{\delta}\left(\top_{x}^{*}\right)=\top_{x}^{*}$.

\section{Example 13}

Let $X$ be a set and $R \in L^{X \times X}$ be an L-fuzzy pre-order. Define $\delta: L^{X} \times L^{X} \rightarrow L$ as

$$
\delta(f, g)=\bigvee_{x, y \in X} R(x, y) \odot f(x) \odot g(y) .
$$

(P1) and (P3) are easily proved.

(P2) For all $f, g \in L^{X}$,

$$
\begin{aligned}
\delta(f, g) & =\bigvee_{x, y \in X} R(x, y) \odot f(x) \odot g(y) \\
& \geq \bigvee_{x \in X} R(x, x) \odot f(x) \odot g(x)=\bigvee_{x \in X} f(x) \odot g(x)
\end{aligned}
$$

(P4) For all $f_{1}, f_{2}, h_{1}, h_{2} \in L^{X}$, by Lemma 2 (17),

$$
\begin{aligned}
\delta\left(f_{1}, h_{1}\right) \oplus \delta\left(f_{2}, h_{2}\right) & =\left(\bigvee_{x, y \in X} R(x, y) \odot f_{1}(x) \odot h_{1}(y)\right) \oplus \\
& \left(\bigvee_{z, w \in X} R(z, w) \odot f_{2}(z) \odot h_{2}(w)\right) \\
& \geq \bigvee_{x, y, z, w \in X}\left(R(x, y) \odot R(z, w) \odot f_{1}(x) \odot f_{2}(z)\right) \odot \\
& \left(h_{1}(y) \oplus h_{2}(w)\right) \\
& \geq \bigvee_{x, y, w \in X}\left(R(x, y) \odot R(y, w) \odot f_{1}(x) \odot f_{2}(x)\right) \odot \\
& \left(h_{1}(w) \oplus h_{2}(w)\right) \\
& =\bigvee_{x, w \in X}\left(\bigvee_{y \in X}(R(x, y) \odot R(y, w))\right. \\
& \odot\left(f_{1}(x) \odot f_{2}(x)\right) \odot\left(h_{1}(w) \oplus h_{2}(w)\right) \\
& =\bigvee_{x, w \in X}\left(R(x, w) \odot f_{1}(x) \odot f_{2}(x)\right) \odot\left(h_{1}(w) \oplus h_{2}(w)\right) \\
& =\delta\left(f_{1} \odot f_{2}, h_{1} \oplus h_{2}\right) .
\end{aligned}
$$

Hence, $\delta$ is an $L$-fuzzy pre-proximity on $X$. Since

$$
\begin{aligned}
\delta(\alpha \odot f, \alpha \rightarrow g) & =\bigvee_{x, y \in X}(R(x, y) \odot(\alpha \odot f)(x) \odot(\alpha \rightarrow g)(y)) \\
& \leq \bigvee_{x, y \in X}(R(x, y) \odot f(x) \odot g(y))=\delta(f, g),
\end{aligned}
$$

$\delta$ is stratified. Moreover, $\delta$ is Alexandrov and generalized. By Theorem 12, we obtain a stratified $L$-fuzzy closure operator $\mathcal{C}_{\delta}: L^{X} \rightarrow L^{X}$ as

$$
\mathcal{C}_{\delta}(f)(x)=\bigwedge_{f \leq g^{*}}\left(g(x) \rightarrow \bigvee_{x, y \in X}\left(R(x, y) \odot g(x) \odot g^{*}(y)\right)\right) .
$$

(1) Let $R=\top_{X \times X}$ be given. Then, $\delta_{1}(f, g)=\bigvee_{x, y \in X} f(x) \odot g(y)$.

Hence, $\delta_{1}$ is an $L$-fuzzy pre-proximity on $X$. Moreover, $\delta_{1}$ is stratified, Alexandrov and generalized. Since $\delta_{1}\left(\top_{x}, \top_{x}^{*}\right)=\top, \delta_{1}$ is not separated.

By Theorem 12, we obtain a stratified $L$-fuzzy closure operator $\mathcal{C}_{\delta_{1}}: L^{X} \rightarrow L^{X}$ as

$$
\mathcal{C}_{\delta_{1}}(f)=\bigwedge_{f \leq g^{*}}\left(g(x) \rightarrow\left(\bigvee_{x, y \in X} g(x) \odot g^{*}(y)\right)\right)
$$

(2) Let $R=\triangle_{X \times X}$ be given, where 


$$
\triangle_{X \times X}(x, y)= \begin{cases}\top, & \text { if } y=x \\ \perp, & \text { otherwise }\end{cases}
$$

Then, $\delta_{2}(f, g)=\bigvee_{x \in X} f(x) \odot g(x)$. Hence, $\delta_{2}$ is an $L$-fuzzy pre-proximity on $X$. Moreover,

(Q) For all $f, g \in L^{X}$,

$$
\begin{aligned}
& \bigwedge_{h \in L^{X}}\left(\delta_{2}(f, h) \oplus \delta_{2}\left(h^{*}, g\right)\right) \\
& =\bigwedge_{h \in L^{X}}\left(\bigvee_{x \in X}(f(x) \odot h(x)) \oplus \bigvee_{x \in X}\left(h^{*}(x) \odot g(x)\right)\right) \quad(\text { Put } h=g) \\
& \leq \bigvee_{x \in X}(f(x) \odot g(x)) \oplus \bigvee_{x \in X}\left(g^{*}(x) \odot g(x)\right) \\
& =\bigvee_{x \in X}(f(x) \odot g(x)) \oplus \perp=\delta_{2}(f, g)
\end{aligned}
$$

Hence, $\delta_{2}$ is an $L$-fuzzy proximity on $X$. Since $\delta_{2}\left(\top_{x}, \top_{x}^{*}\right)=\perp, \delta_{2}$ is separated. Hence, $\delta_{2}$ is separated, stratified, Alexandrov and generalized. By Theorem 12, we obtain a strong, separated, generalized and Alexandrov $L$-fuzzy closure operator $\mathcal{C}_{\delta_{2}}: L^{X} \rightarrow L^{X}$ as follows:

$$
\mathcal{C}_{\delta_{2}}(f)=\bigwedge_{f \leq g^{*}}\left(g(x) \rightarrow\left(\bigvee_{x \in X} g(x) \odot g^{*}(x)\right)\right)=\bigwedge_{f \leq g^{*}}(g(x) \rightarrow \perp)=f .
$$

From the following theorem, we obtain the $L$-fuzzy pre-proximity induced by an $L$-fuzzy closure operator.

Theorem 14 Let $(X, \mathcal{C})$ be an $L$-fuzzy closure space. Define a mapping $\delta_{\mathcal{C}}: L^{X} \times L^{X} \rightarrow L$ by

$$
\delta_{\mathcal{C}}(f, g)=\bigvee_{x \in X} f(x) \odot \mathcal{C}(g)(x) \quad \forall f, g \in L^{X} .
$$

Then, we have the following properties.

(1) $\delta_{\mathcal{C}}$ is an L-fuzzy pre-proximity,

(2) If $\mathcal{C}$ is stratified, then so is $\delta_{\mathcal{C}}$ and $\delta_{\mathcal{C}}(f, \alpha \odot g) \geq \alpha \odot \delta_{\mathcal{C}}(f, g)$,

(3) $\delta_{\mathcal{C}}(f, g) \leq \bigvee_{h \in L^{X}}\left(\delta_{\mathcal{C}}(f, h) \odot \delta_{\mathcal{C}}\left(h^{*}, g\right)\right)$, the equality holds if $\mathcal{C}$ is topological,

(4) If $\mathcal{C}$ is topological, then $\delta_{\mathcal{C}}$ is an L-fuzzy quasi-proximity on $X$,

(5) $\mathcal{C} \leq \mathcal{C}_{\delta_{\mathcal{C}}}$, the equality holds if $\mathcal{C}$ is topological,

(6) If $\mathcal{C}$ is separated, then $\delta_{\mathcal{C}}$ is separated,

(7) $\delta_{\mathcal{C}_{\delta}} \leq \delta$,

(8) If $\mathcal{C}$ is generalized (resp. Alexandrov), then $\delta_{\mathcal{C}}$ is generalized (resp. Alexandrov).

Proof

(1) (P1) Since $\mathcal{C}\left(\perp_{X}\right)=\perp_{X}$ and $\mathcal{C}\left(\top_{X}\right)=\top_{X}$, we have

$$
\begin{aligned}
& \delta_{\mathcal{C}}\left(\top_{X}, \perp_{X}\right)=\bigvee_{x \in X}\left(\top_{X}(x) \odot \mathcal{C}\left(\perp_{X}\right)(x)\right)=\perp, \\
& \delta_{\mathcal{C}}\left(\perp_{X}, \top_{X}\right)=\bigvee_{x \in X}\left(\perp_{X}(x) \odot \mathcal{C}\left(\top_{X}\right)(x)\right)=\perp .
\end{aligned}
$$

(P2) Since $\mathcal{C}(f) \geq f$, we have 


$$
\delta_{\mathcal{C}}(f, g)=\bigvee_{x \in X} f(x) \odot \mathcal{C}(g)(x) \geq \bigvee_{x \in X} f(x) \odot g(x)
$$

(P3) If $f \leq f_{1}$ and $g \leq g_{1}$, then $\mathcal{C}(g) \leq \mathcal{C}\left(g_{1}\right)$. Thus,

$$
\delta_{\mathcal{C}}(f, g)=\bigvee_{x \in X} f(x) \odot \mathcal{C}(g)(x) \leq \bigvee_{x \in X} f_{1}(x) \odot \mathcal{C}\left(g_{1}\right)(x)=\delta_{\mathcal{C}}\left(f_{1}, g_{1}\right) .
$$

(P4)

$$
\begin{aligned}
\delta_{\mathcal{C}}\left(f_{1}, g_{1}\right) \oplus \delta_{\mathcal{C}}\left(f_{2}, g_{2}\right) & =\bigvee_{x \in X}\left(f_{1}(x) \odot \mathcal{C}\left(g_{1}\right)(x)\right) \oplus\left(\bigvee_{x \in X} f_{2}(x) \odot \mathcal{C}\left(g_{2}\right)(x)\right) \\
& \geq \bigvee_{x \in X}\left(f_{1}(x) \odot \mathcal{C}\left(g_{1}\right)(x)\right) \oplus\left(f_{2}(x) \odot \mathcal{C}\left(g_{2}\right)(x)\right) \\
& (\text { by Lemma } 2(13)) \\
& \geq \bigvee_{x \in X}\left(f_{1}(x) \odot f_{2}(x)\right) \odot\left(\mathcal{C}\left(g_{1}\right)(x) \oplus \mathcal{C}\left(g_{2}\right)(x)\right) \\
& \geq \bigvee_{x \in X}\left(f_{1}(x) \odot f_{2}(x)\right) \odot \mathcal{C}\left(g_{1} \oplus g_{2}\right)(x)=\delta_{\mathcal{C}}\left(f_{1} \oplus f_{2}, g_{1} \oplus g_{2}\right) .
\end{aligned}
$$

Hence, $\delta_{\mathcal{C}}$ is an $L$-fuzzy pre-proximity on $X$.

(2) If $\mathcal{C}$ is a stratified, we have

$$
\begin{aligned}
\delta_{\mathcal{C}}(\alpha \odot f, \alpha \rightarrow g) & =\bigvee_{x \in X}(\alpha \odot f)(x) \odot \mathcal{C}(\alpha \rightarrow g)(x) \\
& \leq \bigvee_{x \in X} \alpha \odot f(x) \odot(\alpha \rightarrow \mathcal{C}(g)(x)) \\
& \leq \bigvee_{x \in X} f(x) \odot \mathcal{C}(g)(x)=\delta_{\mathcal{C}}(f, g), \\
& =\bigvee_{x \in X} f(x) \odot \mathcal{C}(\alpha \odot g)(x) \\
& \geq \bigvee_{x \in X} f(x) \odot \alpha \odot \mathcal{C}(g)(x) \\
& =\alpha \odot\left(\bigvee_{x \in X} f(x) \odot \mathcal{C}(g)(x)\right)=\alpha \odot \delta_{\mathcal{C}}(f, g) .
\end{aligned}
$$

(3)

$$
\begin{aligned}
\delta_{\mathcal{C}}^{*}(f, h) \odot \delta_{\mathcal{C}}^{*}\left(h^{*}, g\right) \\
=\left(\bigvee_{x \in X} f(x) \odot \mathcal{C}(h)(x)\right)^{*} \odot\left(\bigvee_{x \in X} h^{*}(x) \odot \mathcal{C}(g)(x)\right)^{*} \\
=\bigwedge_{x \in X}\left(f(x) \rightarrow \mathcal{C}^{*}(h)(x)\right) \odot \bigwedge_{x \in X}\left(h^{*}(x) \rightarrow \mathcal{C}^{*}(g)(x)\right) \\
\quad\left(\operatorname{Since} \mathcal{C}^{*}(h) \leq h^{*}\right) \\
\leq \bigwedge_{x \in X}\left(f(x) \rightarrow h^{*}(x)\right) \odot \bigwedge_{x \in X}\left(h^{*}(x) \rightarrow \mathcal{C}^{*}(g)(x)\right) \\
\leq \bigwedge_{x \in X}\left(f(x) \rightarrow \mathcal{C}^{*}(g)(x)\right)=\delta_{\mathcal{C}}^{*}(f, g) .
\end{aligned}
$$

Hence, $\delta_{\mathcal{C}}(f, g) \leq \bigwedge_{h \in L^{X}}\left(\delta_{\mathcal{C}}(f, h) \oplus \delta_{\mathcal{C}}\left(h^{*}, g\right)\right)$.

If $\mathcal{C}$ is topological, then

$$
\begin{aligned}
& \bigvee_{h \in L^{X}}\left(\delta_{\mathcal{C}}^{*}(f, h) \odot \delta_{\mathcal{C}}^{*}\left(h^{*}, g\right)\right) \\
& =\bigvee_{h \in L^{X}}\left(\bigwedge_{x \in X}\left(f(x) \rightarrow \mathcal{C}^{*}(h)(x)\right)\right) \odot\left(\bigwedge_{x \in X}\left(h^{*}(x) \rightarrow \mathcal{C}^{*}(g)(x)\right)\right) \\
& (\operatorname{put} \mathcal{C}(g)=h) \\
& \geq \bigwedge_{x \in X}\left(f(x) \rightarrow \mathcal{C}^{*}(\mathcal{C}(g))(x)\right) \odot\left(\bigwedge_{x \in X}\left(\mathcal{C}^{*}(g)(x) \rightarrow \mathcal{C}\left(g^{*}\right)(x)\right)\right. \\
& =\bigwedge_{x \in X}\left(f(x) \rightarrow \mathcal{C}^{*}(g)(x)\right)=\delta_{\mathcal{C}}^{*}(f, g) .
\end{aligned}
$$

(4) By (3), it is trivial.

(5) From Lemma 2, we have, 


$$
\begin{aligned}
\mathcal{C}_{\delta_{\mathcal{C}}}(f)(x) & =\bigwedge_{g \in L^{X}}\left\{\bigwedge_{x \in X}\left(g(x) \rightarrow \delta_{\mathcal{C}}\left(g, g^{*}\right)\right) \mid f \leq g^{*}\right\} \\
& =\bigwedge_{g \in L^{X}}\left\{\bigwedge_{x \in X}\left(g(x) \rightarrow\left(\bigvee_{x \in X} g(x) \odot \mathcal{C}\left(g^{*}\right)(x)\right)\right) \mid f \leq g^{*}\right\} \\
& =\left\{\left(\bigvee_{g \in L^{X}} g(x) \odot \bigwedge_{x \in X}\left(\mathcal{C}\left(g^{*}\right)(x) \rightarrow g^{*}(x)\right)\right)^{*} \mid f \leq g^{*}\right\} \\
& \geq\left\{\left(\bigvee_{g \in L^{X}}\left(\bigwedge_{x \in X}\left(\mathcal{C}(f)(x) \rightarrow g^{*}(x)\right) \odot g(x)\right)\right)^{*} \mid \mathcal{C}(f) \leq \mathcal{C}\left(g^{*}\right)\right\} \\
& =\left(\bigvee_{g \in L^{X}}\left(\bigwedge_{x \in X}\left(g(x) \rightarrow \mathcal{C}^{*}(f)(x)\right) \odot g(x)\right)\right)^{*} \geq \mathcal{C}(f)(x) .
\end{aligned}
$$

If $\mathcal{C}$ is topological, then

$$
\begin{aligned}
\mathcal{C}_{\delta_{\mathcal{C}}}(f)(x) & =\bigwedge_{g \in L^{X}}\left\{g(x) \rightarrow \delta_{\mathcal{C}}\left(g, g^{*}\right) \mid f \leq g^{*}\right\} \\
& =\left\{\left(\bigvee_{g \in L^{X}} g(x) \odot \bigwedge_{x \in X}\left(\mathcal{C}\left(g^{*}\right)(x) \rightarrow g^{*}(x)\right)\right)^{*} \mid f \leq g^{*}\right\} \\
& \left(\operatorname{Put} g^{*}=\mathcal{C}(f)\right) \\
& \leq\left(\mathcal{C}^{*}(f)(x) \odot \bigwedge_{x \in X}(\mathcal{C}(\mathcal{C}(f)(x)) \rightarrow \mathcal{C}(f)(x))\right)^{*}=\mathcal{C}(f)(x) .
\end{aligned}
$$

(6) $\delta_{\mathcal{C}_{\delta}}^{*}\left(\top_{x}, \top_{x}^{*}\right)=\bigwedge_{x \in X}\left(\mathcal{C}_{\delta}\left(\top_{x}^{*}\right)(x) \rightarrow \top_{x}^{*}(x)\right)=\top$.

(7)

$$
\begin{aligned}
\delta_{\mathcal{C}_{\delta}}(f, g) & =\bigvee_{x \in X} f(x) \odot \mathcal{C}_{\delta}(g)(x) \\
& =\bigvee_{x \in X} f(x) \odot\left(\bigvee_{h \leq g^{*}} \delta^{*}\left(h, h^{*}\right) \odot h(x)\right)^{*} \\
& \leq \bigvee_{x \in X} f(x) \odot\left(\bigvee_{h \leq g^{*}}\left(\bigwedge_{x \in X}(h(x) \rightarrow h(x)) \odot h(x)\right)\right)^{*} \\
& \leq \bigvee_{x \in X} f(x) \odot g(x) \leq \delta(f, g) .
\end{aligned}
$$

(8) It is easily proved from definitions.

Corollary 15 Let $(X, \mathcal{C})$ be an L-fuzzy closure space. Define a mapping $\delta_{\mathcal{C}}^{S}: L^{X} \times L^{X} \rightarrow L$ by

$$
\delta_{\mathcal{C}}^{s}(f, g)=\bigvee_{x \in X} g(x) \odot \mathcal{C}(f)(x) \quad \forall f, g \in L^{X} .
$$

Then, we have the following properties.

(1) $\delta_{\mathcal{C}}^{s}$ is an L-fuzzy pre-proximity,

(2) If $\mathcal{C}$ is stratified, then $\delta^{s}$ is a stratified,

(3) $\delta_{\mathcal{C}}^{s}(f, g) \leq \bigvee_{h \in L^{X}}\left(\delta_{\mathcal{C}}^{s}(f, h) \odot \delta_{\mathcal{C}}^{s}\left(h^{*}, g\right)\right)$, the equality holds if $\mathcal{C}$ is topological,

(4) If $\mathcal{C}$ is topological, then $\delta_{\mathcal{C}}^{s}$ is a L-fuzzy quasi-proximity on $X$,

(5) $\mathcal{C} \leq \mathcal{C}_{\delta_{\mathcal{C}}^{s}}$, the equality holds if $\mathcal{C}$ is topological,

(6) If $\mathcal{C}$ is separated, then $\delta_{\mathcal{C}}^{s}$ is separated,

(7) $\delta_{\mathcal{C}_{\delta}}^{s} \leq \delta^{s}$,

(8) If $\mathcal{C}$ is generalized (resp. Alexandrov), then $\delta_{\mathcal{C}}^{s}$ is generalized (resp. Alexandrov).

\section{The relationships between $L$-fuzzy pre-proximities and $L$-fuzzy co-topologies}

Theorem 16 Let $\delta$ be an Alexandrov L-fuzzy pre-proximity on X. Define a mapping $\mathcal{F}_{\delta}: L^{X} \rightarrow L$ by $\mathcal{F}_{\delta}(f)=\delta^{*}\left(f^{*}, f\right)$. Then, 
(1) $\mathcal{F}_{\delta}$ is an L-fuzzy co-topology on $X$,

(2) If $\delta$ is stratified, then $\mathcal{F}_{\delta}$ is strong,

(3) If $\delta$ is separated, then $\mathcal{F}_{\delta}$ is separated.

\section{Proof}

(1) $(T 1) \mathcal{F}_{\delta}\left(\perp_{X}\right)=\delta^{*}\left(\perp_{X}^{*}, \perp_{X}\right)=\top, \mathcal{F}_{\delta}\left(\top_{X}\right)=\delta^{*}\left(\top_{X}^{*}, \top_{X}\right)=\top$.

(T2) $\mathcal{F}_{\delta}(f \oplus g)=\delta^{*}\left(f^{*} \odot g^{*}, f \oplus g\right) \geq \delta^{*}\left(f^{*}, f\right) \odot \delta^{*}\left(g^{*}, g\right)=\mathcal{F}_{\delta}(f) \odot \mathcal{F}_{\delta}(g)$.

(T3) $\mathcal{F}_{\delta}\left(\bigwedge_{i \in \Gamma} f_{i}\right)=\delta^{*}\left(\bigvee_{i \in \Gamma} f_{i}^{*}, \bigwedge_{i \in \Gamma} f_{i}\right) \geq \bigwedge_{i \in \Gamma} \delta^{*}\left(f_{i}^{*}, f_{i}\right)=\bigwedge_{i \in \Gamma} \mathcal{F}_{\delta}\left(f_{i}\right)$

(2) $\mathcal{F}_{\delta}(\alpha \odot f)=\delta^{*}\left(\alpha \rightarrow f^{*}, \alpha \odot f\right) \geq \delta^{*}\left(f^{*}, f\right)=\mathcal{F}_{\delta}(f)$,

$$
\mathcal{F}_{\delta}(\alpha \rightarrow f)=\delta^{*}\left(\alpha \odot f^{*}, \alpha \rightarrow f\right) \geq \delta^{*}\left(f^{*}, f\right)=\mathcal{F}_{\delta}(f)
$$

(3) It is easy.

Theorem 17 Let $(X, \mathcal{C})$ be an L-fuzzy closure space. Define the mapping $\mathcal{F}_{\mathcal{C}_{\delta}}: L^{X} \rightarrow L$ by

$$
\mathcal{F}_{\mathcal{C}_{\delta}}(f)=\bigwedge_{x \in X}\left(\mathcal{C}_{\delta}(f)(x) \rightarrow f(x)\right)
$$

Then,

(1) $\mathcal{F}_{\mathcal{C}_{\delta}}$ is an L-fuzzy co-topology on $X$ with $\mathcal{F}_{\mathcal{C}_{\delta}} \geq \mathcal{F}_{\delta}$,

(2) If $\mathcal{C}$ is Alexandrov (resp. strong, separated), then $\mathcal{F}_{\delta_{\mathcal{C}}}$ is Alexandrov (resp. strong, separated).

Proof

(1) (T1) $\mathcal{F}_{\mathcal{C}_{\delta}}\left(\top_{X}\right)=\bigwedge_{x \in X}\left(\mathcal{C}_{\delta}\left(\top_{X}\right)(x) \rightarrow \top_{X}(x)\right)=\top$,

$$
\mathcal{F}_{\mathcal{C}_{\delta}}\left(\perp_{X}\right)=\bigwedge_{x \in X}\left(\mathcal{C}_{\delta}\left(\perp_{X}\right)(x) \rightarrow \perp_{X}(x)\right)=\top .
$$

(T2)

$$
\begin{aligned}
\mathcal{F}_{\mathcal{C}_{\delta}}(f \oplus g) & =\bigwedge_{x \in X}\left(\mathcal{C}_{\delta}(f \oplus g)(x) \rightarrow(f \oplus g)(x)\right) \\
& \geq \bigwedge_{x \in X}\left(\left(\mathcal{C}_{\delta}(f)(x) \oplus \mathcal{C}_{\delta}(g)(x)\right) \rightarrow(f(x) \oplus g(x))\right) \quad \text { (by Lemma 2(13)) } \\
& \geq \bigwedge_{x \in X}\left(\mathcal{C}_{\delta}(f)(x) \rightarrow f(x)\right) \odot \bigwedge_{x \in X}\left(\mathcal{C}_{\delta}(g)(x) \rightarrow g(x)\right) \\
& =\mathcal{F}_{\mathcal{C}_{\delta}}(f) \odot \mathcal{F}_{\mathcal{C}_{\delta}}(g) .
\end{aligned}
$$

(T3) By Lemma 2(16), we have

$$
\begin{aligned}
\mathcal{F}_{\mathcal{C}_{\delta}}\left(\bigwedge_{i \in \Gamma} f_{i}\right) & =\bigwedge_{x \in X}\left(\mathcal{C}_{\delta}\left(\bigwedge_{i \in \Gamma} f_{i}\right)(x) \rightarrow\left(\bigwedge_{i \in \Gamma} f_{i}\right)(x)\right) \\
& \geq \bigwedge_{x \in X}\left(\bigwedge_{i \in \Gamma} \mathcal{C}_{\delta}\left(f_{i}\right)(x) \rightarrow \bigwedge_{i \in \Gamma} f_{i}(x)\right) \\
& \geq \bigwedge_{i \in \Gamma}\left(\bigwedge_{x \in X}\left(\mathcal{C}_{\delta}\left(f_{i}\right)(x) \rightarrow f_{i}(x)\right)\right)=\bigwedge_{i \in \Gamma} \mathcal{F}_{\mathcal{C}_{\delta}}\left(f_{i}\right) .
\end{aligned}
$$


Hence, $\mathcal{F}_{\mathcal{C}_{\delta}}$ is an $L$-fuzzy co-topology on $X$.

Moreover,

$$
\begin{aligned}
\mathcal{F}_{\mathcal{C}_{\delta}}(f) & =\bigwedge_{x \in X}\left(\mathcal{C}_{\delta}(f)(x) \rightarrow f(x)\right) \\
& =\left(\bigvee_{x \in X} \mathcal{C}_{\delta}(f)(x) \odot f^{*}(x)\right)^{*} \\
& \geq\left(\bigvee_{x \in X} f(x) \odot f^{*}(x)\right)^{*} \geq \delta^{*}\left(f^{*}, f\right)=\mathcal{F}_{\delta}(f)
\end{aligned}
$$

(2)

$$
\begin{aligned}
\mathcal{F}_{\mathcal{C}_{\delta}}\left(\bigvee_{i \in \Gamma} f_{i}\right) & =\bigwedge_{x \in X}\left(\mathcal{C}_{\delta}\left(\bigvee_{i \in \Gamma} f_{i}\right)(x) \rightarrow \bigvee_{i \in \Gamma} f_{i}(x)\right) \\
& \left.=\bigwedge_{x \in X}\left(\bigvee_{i \in \Gamma} \mathcal{C}_{\delta}\left(f_{i}\right)(x) \rightarrow \bigvee_{i \in \Gamma} f_{i}(x)\right)\right) \\
& \geq \bigwedge_{i \in \Gamma}\left(\bigwedge_{x \in X}\left(\mathcal{C}_{\delta}\left(f_{i}\right)(x) \rightarrow f_{i}(x)\right)\right)=\bigwedge_{i \in \Gamma} \mathcal{F}_{\mathcal{C}_{\delta}}\left(f_{i}\right)
\end{aligned}
$$

Hence, $\mathcal{F}_{\mathcal{C}_{\delta}}$ is an Alexandrov $L$-fuzzy co-topology on $X$. By Lemma 2(14)(18), we have

$$
\begin{aligned}
\mathcal{F}_{\mathcal{C}_{\delta}}(\alpha \odot f) & =\bigwedge_{x \in X}\left(\mathcal{C}_{\delta}(\alpha \odot f)(x) \rightarrow(\alpha \odot f(x))\right) \\
& \geq \bigwedge_{x \in X}\left(\left(\alpha \odot \mathcal{C}_{\delta}(f)(x)\right) \rightarrow(\alpha \odot f(x))\right) \\
& \geq \bigwedge_{x \in X}\left(f(x) \rightarrow \mathcal{C}_{\delta}(f)(x)\right)=\mathcal{F}_{\mathcal{C}_{\delta}}(f), \\
\mathcal{F}_{\mathcal{C}_{\delta}}(\alpha \rightarrow f) & =\bigwedge_{x \in X}\left(\mathcal{C}_{\delta}(\alpha \rightarrow f)(x) \rightarrow(\alpha \rightarrow f(x))\right) \\
& \geq \bigwedge_{x \in X}\left(\left(\alpha \rightarrow \mathcal{C}_{\delta}(f)(x)\right) \rightarrow(\alpha \rightarrow f(x))\right) \\
& \geq \bigwedge_{x \in X}\left(\mathcal{C}_{\delta}(f)(x) \rightarrow f(x)\right)=\mathcal{F}_{\mathcal{C}_{\delta}}(f) .
\end{aligned}
$$

Other cases are easily proved.

Theorem 18 Let $(X, \delta)$ be an L-fuzzy pre-proximity space. Then, the mapping $\mathcal{F}_{\delta}^{(1)}: L^{X} \rightarrow$ L defined by $\mathcal{F}_{\delta}^{(1)}(f)=\bigwedge_{x \in X}\left(\delta\left(f, \top_{x}\right) \rightarrow f(x)\right)$ is an L-fuzzy co-topology on $X$. Moreover, if $\delta$ is Alexandrov and $\delta(\alpha \odot f, g) \geq \alpha \odot \delta(f, g)$, then $\mathcal{F}_{\delta}^{(1)}\left(f^{*}\right) \geq \mathcal{F}_{\delta}(f)$.

Proof

(1) (T1) $\mathcal{F}_{\delta}^{(1)}\left(\perp_{X}\right)=\bigwedge_{x \in X}\left(\delta\left(\perp_{X}, \top_{x}\right) \rightarrow \perp_{X}(x)\right)=\top$,

$$
\mathcal{F}_{\delta}^{(1)}\left(\top_{x}\right)=\bigwedge_{x \in X}\left(\delta\left(\top_{X}, \top_{x}\right) \rightarrow \top_{X}(x)\right)=\top .
$$

(T2)

$$
\begin{aligned}
\mathcal{F}_{\delta}^{(1)}(f \oplus g) & =\bigwedge_{x \in X}\left(\delta\left(f \oplus g, \top_{x}\right) \rightarrow(f \oplus g)(x)\right) \\
& \geq \bigwedge_{x \in X}\left(\left(\delta\left(f, \top_{x}\right) \oplus \delta\left(g, \top_{x}\right)\right) \rightarrow(f(x) \oplus g(x))\right) \\
& \geq \bigwedge_{x \in X}\left(\delta\left(f, \top_{x}\right) \rightarrow f(x)\right) \odot \bigwedge_{x \in X}\left(\delta\left(g, \top_{x}\right) \rightarrow g(x)\right) \\
& \geq \mathcal{F}_{\delta}^{(1)}(f) \odot \mathcal{F}_{\delta}^{(1)}(g) .
\end{aligned}
$$

(T3)

$$
\begin{aligned}
\mathcal{F}_{\delta}^{(1)}\left(\bigwedge_{i \in \Gamma} f_{i}\right) & =\bigwedge_{x \in X}\left(\delta\left(\bigwedge_{i \in \Gamma} f_{i}, \top_{x}\right) \rightarrow \bigwedge_{i \in \Gamma} f_{i}(x)\right) \\
& =\bigwedge_{i \in \Gamma} \bigwedge_{x \in X}\left(\delta\left(\bigwedge_{i \in \Gamma} f_{i}, \top_{x}\right) \rightarrow f_{i}(x)\right) \\
& \geq \bigwedge_{i \in \Gamma} \bigwedge_{x \in X}\left(\delta\left(f_{i}, \top_{x}\right) \rightarrow f_{i}(x)\right)=\bigwedge_{i \in \Gamma} \mathcal{F}_{\delta}^{(1)}\left(f_{i}\right)
\end{aligned}
$$

Moreover, if $\delta$ is Alexandrov, then 


$$
\begin{aligned}
\mathcal{F}_{\delta}^{(1)}\left(\bigvee_{i \in \Gamma} f_{i}\right) & =\bigwedge_{x \in X}\left(\delta\left(\bigvee_{i \in \Gamma} f_{i}, \top_{x}\right) \rightarrow \bigvee_{i \in \Gamma} f_{i}(x)\right) \\
& =\bigwedge_{x \in X}\left(\bigvee_{i \in \Gamma} \delta\left(f_{i}, \top_{x}\right) \rightarrow \bigvee_{i \in \Gamma} f_{i}(x)\right) \\
& \geq \bigwedge_{i \in \Gamma} \bigwedge_{x \in X}\left(\delta\left(f_{i}, \top_{x}\right) \rightarrow f_{i}(x)\right)=\bigwedge_{i \in \Gamma} \mathcal{F}_{\delta}^{(1)}\left(f_{i}\right)
\end{aligned}
$$

Hence, $\mathcal{F}_{\delta}^{(1)}$ is Alexandrov $L$-fuzzy co-topology on $X$.

If $\delta(\alpha \odot f, g) \geq \alpha \odot \delta(f, g)$, then

$$
\begin{aligned}
\mathcal{F}_{\delta}(f)=\delta^{*}\left(f^{*}, f\right) & =\delta^{*}\left(f^{*}, \bigvee_{x \in X} f(x) \odot \top_{x}\right) \\
& \leq \bigwedge_{x \in X}\left(f(x) \rightarrow \delta^{*}\left(f^{*}, \top_{x}\right)\right) \\
& =\bigwedge_{x \in X}\left(\delta\left(f^{*}, \top_{x}\right) \rightarrow f^{*}(x)\right)=\mathcal{F}_{\delta}^{(1)}\left(f^{*}\right)
\end{aligned}
$$

\section{Example 19}

Let $X=\left\{h_{i} \mid i=\{1, \ldots, 3\}\right\}$ with $h_{i}=$ house and $Y=\{e, b, w, c, i\}$ with $e=$ expensive, $b=$ beautiful, $w=$ wooden, $c=$ creative, $i=$ in the green surroundings. Let $\left([0,1], \odot, \rightarrow^{*}, 0,1\right)$ be a complete residuated lattice as

$$
x \odot y=\max \{0, x+y-1\}, x \rightarrow y=\min \{1-x+y, 1\}, x^{*}=1-x .
$$

Let $I \in[0,1]^{X \times Y}$ be a fuzzy information as follows:

$$
\begin{array}{cccccc}
I & e & b & w & c & i \\
h_{1} & 0.7 & 0.6 & 0.5 & 0.9 & 0.2 \\
h_{2} & 0.6 & 0.8 & 0.4 & 0.3 & 0.5 \\
h_{3} & 0.4 & 0.9 & 0.8 & 0.6 & 0.6
\end{array}
$$

Define [0, 1]-fuzzy pre-orders $R_{X}^{Y}, R_{X}^{\{b, w\}} \in[0,1]^{X \times X}$ by

$$
\begin{gathered}
R_{X}^{Y}\left(h_{i}, h_{j}\right)=\bigwedge_{y \in Y}\left(I\left(h_{i}, y\right) \rightarrow I\left(h_{j}, y\right)\right), \\
R_{X}^{b, w\}}\left(h_{i}, h_{j}\right)=\bigwedge_{y \in\{b, w\}}\left(R\left(h_{i}, y\right) \rightarrow R\left(h_{j}, y\right)\right), \\
R_{X}^{Y}=\left(\begin{array}{ccc}
1 & 0.4 & 0.7 \\
0.7 & 1 & 0.8 \\
0.6 & 0.6 & 1
\end{array}\right), R_{X}^{\{b, w\}}=\left(\begin{array}{ccc}
1 & 0.9 & 1 \\
0.8 & 1 & 1 \\
0.7 & 0.6 & 1
\end{array}\right) .
\end{gathered}
$$

(1) For each $R \in\left\{R_{X}^{Y}, R_{X}^{\{b, w\}}\right\}$, by Example 13, we obtain a stratified, Alexandrov and generalized $[0,1]$-fuzzy pre-proximity $\delta_{R}:[0,1]^{X} \times[0,1]^{X} \rightarrow[0,1]$ as

$$
\delta_{R}(f, g)=\bigvee_{h_{i}, h_{j} \in X} R_{X}^{Y}\left(h_{i}, h_{j}\right) \odot f\left(h_{i}\right) \odot g\left(h_{j}\right) .
$$

By Theorem 12, we obtain a stratified $[0,1]$-fuzzy closure operator $C_{\delta_{R}}:[0,1]^{X} \rightarrow[0,1]^{X}$ as

$$
\begin{aligned}
C_{\delta_{R}}(f)\left(h_{i}\right) & =\bigwedge_{g \in L^{X}}\left(\left(S\left(f, g^{*}\right) \odot g\left(h_{i}\right)\right) \rightarrow \delta_{R}\left(g, g^{*}\right)\right) \\
& =\bigwedge_{g \in L^{X}}\left(\left(S\left(f, g^{*}\right) \odot g\left(h_{i}\right)\right)\right. \\
& \left.\rightarrow\left(\bigvee_{h_{j}, h_{k} \in X} R_{X}^{Y}\left(h_{j}, h_{k}\right) \odot g\left(h_{j}\right) \odot g^{*}\left(h_{k}\right)\right)\right) .
\end{aligned}
$$

By Theorem 16, we obtain a strong $[0,1]$-fuzzy co-topology $\mathcal{F}_{\delta_{R}}:[0,1]^{X} \rightarrow[0,1]$ as 


$$
\begin{aligned}
\mathcal{F}_{\delta_{R}}(f)=\delta_{R}^{*}\left(f^{*}, f\right) & =\left(\bigvee_{h_{i}, h_{j} \in X} R_{X}^{Y}\left(h_{i}, h_{j}\right) \odot f^{*}\left(h_{i}\right) \odot f\left(h_{j}\right)\right)^{*} \\
& =\bigwedge_{h_{i}, h_{j} \in X}\left(R_{X}^{Y}\left(h_{i}, h_{j}\right) \odot f\left(h_{j}\right) \rightarrow f\left(h_{i}\right)\right) .
\end{aligned}
$$

Since

$$
\delta_{R}\left(f, \top_{h_{j}}\right)=\bigvee_{h_{i}, h_{j} \in X} R_{X}^{Y}\left(h_{i}, h_{j}\right) \odot f\left(h_{i}\right) \odot \top_{h_{j}}\left(h_{j}\right)=\bigvee_{h_{i} \in X} R\left(h_{i}, h_{j}\right) \odot f\left(h_{i}\right),
$$

by Theorem 18, we obtain $[0,1]$-fuzzy co-topology $\mathcal{F}_{\delta_{R}}^{(1)}:[0,1]^{X} \rightarrow[0,1]$ as

$$
\begin{aligned}
\mathcal{F}_{\delta_{R}}^{(1)}(f) & =\bigwedge_{h_{j} \in X}\left(\delta_{R}\left(f, \top_{h_{j}}\right) \rightarrow f\left(h_{j}\right)\right) \\
& =\bigwedge_{h_{j} \in X}\left(\left(\bigvee_{h_{i} \in X} R\left(h_{i}, h_{j}\right) \odot f\left(h_{i}\right)\right) \rightarrow f\left(h_{j}\right)\right) \\
& =\bigwedge_{h_{i}, h_{j} \in X}\left(\left(R\left(h_{i}, h_{j}\right) \odot f\left(h_{i}\right)\right) \rightarrow f\left(h_{j}\right)\right) .
\end{aligned}
$$

(2) For each $R \in\left\{R_{X}^{Y}, R_{X}^{\{b, w\}}\right\}$, we obtain a strong, generalized, topological and Alexandrov $[0,1]$-fuzzy closure operator $C_{R}:[0,1]^{X} \rightarrow[0,1]^{X}$ as

$$
C_{R}(f)\left(h_{j}\right)=\bigvee_{h_{i} \in X} R\left(h_{i}, h_{j}\right) \odot f\left(h_{i}\right) .
$$

By Theorem 14, we obtain a generalized, topological and Alexandrov [0, 1]-fuzzy quasiproximity $\delta_{C_{R}}$ as

$$
\begin{aligned}
\delta_{C_{R}}(f, g) & =\bigvee_{h_{i} \in X} f\left(h_{i}\right) \odot C_{R}(g)\left(h_{i}\right) \\
& =\bigvee_{h_{i} \in X} f\left(h_{i}\right) \odot\left(\bigvee_{h_{j} \in X} R\left(h_{j}, h_{i}\right) \odot g\left(h_{j}\right)\right) \\
& =\bigvee_{h_{i}, h_{j} \in X} R\left(h_{j}, h_{i}\right) \odot f\left(h_{i}\right) \odot g\left(h_{j}\right) .
\end{aligned}
$$

By Theorem 16, we obtain [0, 1]-fuzzy co-topologies $\mathcal{F}_{\delta_{C_{R}}}$ and $\mathcal{F}_{\delta_{C_{R}}}^{(1)}$ as follows:

$$
\begin{aligned}
\mathcal{F}_{\delta_{C_{R}}}(f)=\delta_{C_{R}}^{*}\left(f^{*}, f\right) & =\left(\bigvee_{h_{i}, h_{j} \in X} R\left(h_{i}, h_{j}\right) \odot f^{*}\left(h_{i}\right) \odot f\left(h_{j}\right)\right)^{*} \\
& =\bigwedge_{h_{i}, h_{j} \in X}\left(R_{X}^{Y}\left(h_{i}, h_{j}\right) \odot f\left(h_{j}\right) \rightarrow f\left(h_{i}\right)\right) .
\end{aligned}
$$

Also we have

$$
\begin{aligned}
\mathcal{F}_{\delta_{C_{R}}^{(1)}(f)} & =\bigwedge_{h_{j} \in X}\left(\delta_{R}\left(f, \top_{h_{j}}\right) \rightarrow f\left(h_{j}\right)\right) \\
& =\bigwedge_{h_{j} \in X}\left(\left(\bigvee_{h_{i} \in X} R\left(h_{i}, h_{j}\right) \odot f\left(h_{i}\right)\right) \rightarrow f\left(h_{j}\right)\right) \\
& =\bigwedge_{h_{i}, h_{j} \in X}\left(\left(R\left(h_{i}, h_{j}\right) \odot f\left(h_{i}\right)\right) \rightarrow f\left(h_{j}\right)\right) .
\end{aligned}
$$

\section{Galois correspondences}

Theorem 20 Let $\varphi: X \rightarrow Y$ be a mapping. Then

(1) $D_{\delta}(\varphi) \leq D_{\mathcal{C}_{\delta}}(\varphi)$

(2) $D_{\delta}(\varphi)=D_{\mathcal{F}_{\delta}}(\varphi)$

(3) $D_{\delta}(\varphi) \leq D_{\mathcal{F}_{\delta}}(1)(\varphi)$.

Proof

(1) By Lemma 2(18), we have 


$$
\begin{aligned}
D_{\mathcal{C}_{\delta}}(\varphi) & =\bigwedge_{f \in L^{Y}} \bigwedge_{x \in X}\left(\mathcal{C}_{\delta_{X}}\left(\varphi^{\leftarrow}(f)\right)(x) \rightarrow \varphi^{\leftarrow}\left(\mathcal{C}_{\delta_{Y}}(f)\right)(x)\right) \\
& =\bigwedge_{f \in L^{Y}} \bigwedge_{x \in X}\left(\bigwedge_{h \in L^{X}}\left\{h(x) \rightarrow \delta_{X}\left(h, h^{*}\right) \mid \varphi^{\leftarrow}(f) \leq h^{*}\right\}\right. \\
& \left.\rightarrow \bigwedge_{g \in L^{Y}}\left\{g(y) \rightarrow \delta_{Y}\left(g, g^{*}\right) \mid f \leq g^{*}\right\}\right) \\
& \geq \bigwedge_{f \in L^{Y}} \bigwedge_{x \in X}\left(\bigwedge_{h \in L^{X}}\left\{h(x) \rightarrow \delta_{X}\left(h, h^{*}\right) \mid \varphi^{\leftarrow}(f) \leq h^{*}\right\}\right. \\
& \left.\rightarrow \bigwedge_{g \in L^{Y}}\left\{g(\varphi(x)) \rightarrow \delta_{Y}\left(g, g^{*}\right) \mid \varphi^{\leftarrow}(f) \leq \varphi^{\leftarrow}\left(g^{*}\right)\right\}\right) \\
& \geq \bigwedge_{f \in L^{Y}} \bigwedge_{x \in X}\left(\left(\varphi^{\leftarrow}(g)(x) \rightarrow \delta_{X}\left(\varphi^{\leftarrow}(g), \varphi^{\leftarrow}\left(g^{*}\right)\right)\right) \rightarrow\left(\varphi^{\leftarrow}(g)(x) \rightarrow \delta_{Y}\left(g, g^{*}\right)\right)\right) \\
& \geq \bigwedge_{f, g \in L^{Y}}\left(\delta_{X}\left(\varphi^{\leftarrow}(g), \varphi^{\leftarrow}\left(g^{*}\right)\right) \rightarrow \delta_{Y}\left(g, g^{*}\right)\right)=D_{\delta}(\varphi) .
\end{aligned}
$$

(2)

$$
\begin{aligned}
D_{\mathcal{F}_{\delta}}(\varphi) & =\bigwedge_{f \in L^{Y}}\left(\mathcal{F}_{\delta_{Y}}(f) \rightarrow \mathcal{F}_{\delta_{X}}\left(\varphi^{\leftarrow}(f)\right)\right) \\
& =\bigwedge_{f \in L^{Y}}\left(\delta_{Y}^{*}\left(f^{*}, f\right) \rightarrow \delta_{X}^{*}\left(\varphi^{\leftarrow}\left(f^{*}\right), \varphi^{\leftarrow}(f)\right)\right) \\
& \left.=\bigwedge_{f \in L^{Y}}\left(\delta_{X}\left(\varphi^{\leftarrow}\left(f^{*}\right), \varphi^{\leftarrow}(f)\right) \rightarrow \delta_{Y}\left(f^{*}, f\right)\right)\right)=D_{\delta}(\varphi) .
\end{aligned}
$$

(3)

$$
\begin{aligned}
D_{\mathcal{F}_{\delta}^{(1)}}(\varphi) & =\bigwedge_{f \in L^{Y}}\left(\mathcal{F}_{\delta_{Y}}^{(1)}(f) \rightarrow \mathcal{F}_{\delta_{X}}^{(1)}\left(\varphi^{\leftarrow}(f)\right)\right) \\
& =\bigwedge_{f \in L^{Y}}\left(\bigwedge_{y \in Y}\left(\delta_{Y}\left(f, \top_{y}\right) \rightarrow f(y)\right)\right. \\
& \rightarrow \bigwedge_{x \in X}\left(\delta_{X}\left(\varphi^{\leftarrow}(f), \varphi^{\leftarrow}\left(\top_{\varphi(x)}\right) \rightarrow \varphi^{\leftarrow}(f)(x)\right)\right) \\
& \geq \bigwedge_{f \in L^{Y}}\left(\bigwedge_{x \in X}\left(\delta_{Y}\left(f, \top_{\varphi(x)}\right) \rightarrow f(\varphi(x))\right)\right. \\
& \rightarrow \bigwedge_{x \in X}\left(\delta_{X}\left(\varphi^{\leftarrow}(f), \varphi^{\leftarrow}\left(\top_{\varphi(x)}\right) \rightarrow f(\varphi(x))\right)\right. \\
& \geq \bigwedge_{f \in L^{Y}} \bigwedge_{x \in X}\left(\delta_{X}\left(\varphi^{\leftarrow}(f), \varphi^{\leftarrow}\left(\top_{\varphi(x)}\right) \rightarrow \delta_{Y}\left(f, \top_{\varphi(x)}\right)\right)\right. \\
& =\bigwedge_{f \in L^{Y}}\left(\delta_{X}\left(\varphi^{\leftarrow}(f), \varphi^{\leftarrow}\left(\top_{\varphi(x)}\right) \rightarrow \delta_{Y}\left(f, \top_{\varphi(x)}\right)\right)=D_{\delta}(\varphi) .\right.
\end{aligned}
$$

Theorem 21 Let $\varphi: X \rightarrow Y$ be a mapping. Then,

(1) $D_{\mathcal{C}}(\varphi) \leq D_{\delta_{\mathcal{C}}}(\varphi)$

(2) $D_{\mathcal{C}_{\delta}}(\varphi) \leq D_{\mathcal{F}_{\delta}}(\varphi)$.

\section{Proof}

(1) From Lemma 2(18), we have

$$
\begin{aligned}
D_{\delta_{\mathcal{C}}}(\varphi) & =\bigwedge_{f, g \in L^{Y}}\left(\delta_{\left.\mathcal{C}_{X}\left(\varphi^{\leftarrow}(f), \phi^{\leftarrow}(g)\right) \rightarrow \delta_{\mathcal{C}_{Y}}(f, g)\right)}\right. \\
& =\bigwedge_{f, g \in L^{Y}}\left(\bigvee_{x \in X} \varphi^{\leftarrow}(f)(x) \odot \mathcal{C}_{X}\left(\varphi^{\leftarrow}(g)\right)(x) \rightarrow \bigvee_{y \in Y} f(y) \odot \mathcal{C}_{Y}(g)(y)\right) \\
& \geq \bigwedge_{f, g \in L^{Y}}\left(\bigvee_{x \in X} f(\varphi(x)) \odot \mathcal{C}_{X}\left(\varphi^{\leftarrow}(g)\right)(x) \rightarrow \bigvee_{x \in X} f(\varphi(x)) \odot \mathcal{C}_{Y}(g)(\varphi(x))\right) \\
& \geq \bigwedge_{g \in L^{Y}} \bigwedge_{x \in X}\left(\mathcal{C}_{X}\left(\varphi^{\leftarrow}(g)\right)(x) \rightarrow \varphi^{\leftarrow}\left(\mathcal{C}_{Y}(g)\right)(x)\right)=D_{\mathcal{C}}(\varphi) .
\end{aligned}
$$

(2) From Lemma 2(18), we have 


$$
\begin{aligned}
D_{\mathcal{F}_{\mathcal{C}_{\delta}}}(\varphi) & =\bigwedge_{f \in L^{Y}}\left(\mathcal{F}_{\mathcal{C}_{\delta_{Y}}}(f) \rightarrow \mathcal{F}_{\mathcal{C}_{\delta_{X}}}\left(\varphi^{\leftarrow}(f)\right)\right) \\
& =\bigwedge_{f \in L^{Y}}\left(\bigwedge_{y \in Y}\left(\mathcal{C}_{\delta_{Y}}(f)(y) \rightarrow f(y)\right)\right. \\
& \left.\rightarrow \bigwedge_{x \in X}\left(\mathcal{C}_{\delta_{X}}\left(\varphi^{\leftarrow}(f)\right)(x) \rightarrow \varphi^{\leftarrow}(f)(x)\right)\right) \\
& \geq \bigwedge_{f \in L^{Y}}\left(\bigwedge_{x \in X}\left(\mathcal{C}_{\delta_{Y}}(f)(\varphi(x)) \rightarrow f(\varphi(x))\right)\right. \\
& \left.\rightarrow \bigwedge_{x \in X}\left(\mathcal{C}_{\delta_{X}}\left(\varphi^{\leftarrow}(f)\right)(x) \rightarrow \varphi^{\leftarrow}(f)(x)\right)\right) \\
& =\bigwedge_{f \in L^{Y}}\left(\bigwedge_{x \in X}\left(\varphi^{\leftarrow}\left(\mathcal{C}_{\delta_{Y}}(f)\right)(x) \rightarrow \varphi^{\leftarrow}(f)(x)\right)\right. \\
& \left.\rightarrow \bigwedge_{x \in X}\left(\mathcal{C}_{\delta_{X}}\left(\varphi^{\leftarrow}(f)\right)(x) \rightarrow \varphi^{\leftarrow}(f)(x)\right)\right) \\
& \geq \bigwedge_{f \in L^{Y}} \bigwedge_{x \in X}\left(\mathcal{C}_{\delta_{X}}\left(\varphi^{\leftarrow}(f)\right)(x) \rightarrow \varphi^{\leftarrow}\left(\mathcal{C}_{\delta_{Y}}(f)\right)(x)\right)=D_{\mathcal{C}_{\delta}}(\varphi)
\end{aligned}
$$

Definition 22 [40] Suppose that $F: \mathcal{D} \rightarrow \mathcal{C}, G: \mathcal{C} \rightarrow \mathcal{D}$ are concrete functors. The pair $(F, G)$ is called a Galois correspondence between $\mathcal{C}$ and $\mathcal{D}$ if for each $Y \in \mathcal{C}$, $i d_{Y}: F \circ G(Y) \rightarrow Y$ is a $\mathcal{C}$-morphism, and for each $X \in \mathcal{D}$, id $X: X \rightarrow G \circ F(X)$ is a $\mathcal{D}$ -morphism.

If $(F, G)$ is a Galois correspondence, then it is easy to check that $F$ is a left adjoint of $G$, or equivalently that $G$ is a right adjoint of $F$.

The category of separated $L$-fuzzy pre-proximity spaces with $L F$-proximity mappings as morphisms is denoted by SPROX.

The category of separated $L F$-fuzzy closure spaces with $L F$-closure mappings as morphisms is denoted by SFC.

From Theorems 12 and 20, we obtain a concrete functor $\Theta:$ SPROX $\rightarrow$ SFC defined as

$$
\Theta(X, \delta)=\left(X, \mathcal{C}_{\delta}\right), \Theta(\phi)=\phi .
$$

From Theorems 14 and 21, we obtain a concrete functor $\Gamma: \mathbf{S F C} \rightarrow \operatorname{SPROX}$ defined as

$$
\Gamma(X, \mathcal{C})=\left(X, \delta_{\mathcal{C}}\right), \Gamma(\phi)=\phi .
$$

Theorem $23 \Gamma: \mathbf{S F C} \rightarrow$ SPROX is a left adjoint of $\Theta: \mathbf{S P R O X} \rightarrow \mathbf{S F C}$, i.e, $(\Theta, \Gamma)$ is a Galois correspondence.

\section{Proof}

By Theorem 14(5), if $\mathcal{C}_{X}$ is a separated L-fuzzy closure operator on a set $X$, then $\Theta\left(\Gamma\left(C_{X}\right)\right)=\mathcal{C}_{\delta_{\mathcal{C}_{X}}} \geq \mathcal{C}_{X}$. Hence, the identity map id $d_{X}:\left(X, \mathcal{C}_{X}\right) \rightarrow\left(X, \mathcal{C}_{\delta_{\mathcal{C}_{X}}}\right)=\left(X, \Theta\left(\Gamma\left(C_{X}\right)\right)\right)$ is an LF-closure map. Moreover, if $\delta_{Y}$ is a separated L-fuzzy pre-proximity on a set $Y$, by Theorem 14(7), we have $\Gamma\left(\Theta\left(\delta_{Y}\right)\right)=\delta_{\mathcal{C}_{\delta_{Y}}} \leq \delta_{Y}$. Hence, the identity map id ${ }_{Y}:\left(Y, \Gamma\left(\Theta\left(\delta_{Y}\right)\right)\right) \rightarrow\left(Y, \delta_{Y}\right)$ is an LFproximity map. Therefore, $(\Theta, \Gamma)$ is a Galois correspondence. 


\section{Conclusion.}

In this paper, $L$-fuzzy pre-proximities and $L$-fuzzy closure operators in complete residuated lattice are investigated. From a given $L$-fuzzy pre-proximity $\delta$, we can obtain an $L$-fuzzy closure operator $\mathcal{C}_{\delta}$ (see Theorem 12). Conversely, for given $L$-fuzzy closure space $\mathcal{C}$, we obtain $L$-fuzzy pre-proximity $\delta_{\mathcal{C}}$ (see Theorem 14) and $L$-fuzzy co-topologies $\mathcal{F}_{\delta}$ and $\mathcal{F}_{\mathcal{C}_{\delta}}$ (Theorems $16,17,18$ ). It is also shown that there is a Galois correspondence between the category of (separated) $L$-fuzzy closure spaces and that of (separated) $L$-fuzzy pre-proximity spaces (Theorem 21 ). We give Example 19 as a viewpoint of the topological structure for fuzzy information and fuzzy rough sets in a complete residuated lattice.

In the future, the concepts of $L$-fuzzy pre-proximity spaces, information systems and decision rules with a view point of applications to multi-attribute decision-making will be investigated in residuated lattices.

\section{Acknowledgements}

Not applicable.

Authors' contributions

All authors jointly worked on the results, and they read and approved the final manuscript.

Funding

Not applicable.

Availability of data and materials

Not applicable.

Declarations

Competing interests

The authors declare that they have no competing interests.

Received: 21 November 2019 Accepted: 15 February 2021

Published online: 24 March 2021

\section{References}

1. Goguen, J.A.: L-fuzzy sets. J. Math. Anal. Appl. 18, 145-174 (1967)

2. Zadeh, L.A.: Fuzzy sets. Inf. Control 8, 338-353 (1965)

3. Bandler, W., Kohout, L.: Special properties, closures and interiors of crisp and fuzzy relations. Fuzzy Sets Syst. 26(3), 317-331 (1988)

4. Biacino, L., Gerla, G.: Closure systems and L-subalgebras. Inf. Sci. 33, 181-195 (1984)

5. Biacino, L., Gerla, G.: An extension principle for closure operators. J. Math. Anal. Appl. 198, 1-24 (1996)

6. Bodenhofer, U., De Cock, M., Kerre, E.E.: Openings and closures of fuzzy preorderings: theoretical basics and applications to fuzzy rule-based systems. Int. J. Gen. Syst. 32(4), 343-360 (2003)

7. Ward, M., Dilworth, R.P.: Residuated lattices. Trans. Am. Math. Soc. 45, 335-354 (1939)

8. Bělohlávek, R.: Fuzzy Relational Systems. Kluwer Academic Publishers, New York (2002)

9. Höhle, U., Rodabaugh, S.E.: Mathematics of Fuzzy Sets: Logic, Topology, and Measure Theory. The Handbooks of Fuzzy Sets Series 3. Kluwer Academic Publishers, Boston (1999)

10. Rodabaugh, S.E., Klement, E.P.: Topological and Algebraic Structures in Fuzzy Sets. The Handbook of Recent Developments in the Mathematics of Fuzzy Sets. Kluwer Academic Publishers, Boston (2003)

11. Turunen, E.: Mathematics Behind Fuzzy Logic. Springer, Heidelberg (1999)

12. Zhang, D.: An enriched category approach to many valued topology. Fuzzy Sets Syst. 158, 349-366 (2007)

13. Bělohlávek, R.: Fuzzy closure operators. J. Math. Anal. Appl. 262, 473-489 (2001)

14. Bělohlávek, R.: Fuzzy closure operators II. Soft Comput. 7(1), 53-64 (2002)

15. Fang, J., Yue, Y.: L-fuzzy closure systems. Fuzzy Sets Syst. 161, 1242-1252 (2010)

16. Shi, F.G., Pang, B.: Categories isomorphic to the category of L-fuzzy closure system spaces. Iran. J. Fuzzy Syst. 10, 127-146 (2013)

17. Ramadan, A.A.: L-fuzzy interior systems. Comput. Math. Appl. 62, 4301-4307 (2011)

18. Katsaras, A.K., Petalas, C.G.: A unified theory of fuzzy topologies, fuzzy proximities and fuzzy uniformities. Rev. Roum. Math. Pures Appl. 28, 845-896 (1983)

19. Katsaras, A.K.: Fuzzy syntopogenous structures compatible with Lowen fuzzy uniformities and Artico-Moresco fuzzy proximities. Fuzzy Sets Syst. 36, 375-393 (1990)

20. Chang, C.L.: Fuzzy topological spaces. J. Math. Anal. Appl. 24, 182-190 (1968) 
21. Katsaras, A.K.: Fuzzy proximity spaces. J. Math. Anal. Appl. 68, 100-110 (1979)

22. Hutton, B.: Uniformities in fuzzy topological spaces. J. Math. Anal. Appl. 58, 74-79 (1977)

23. Bayoumi, F.: On L-proximities of the internal type. Fuzzy Sets Syst. 157, 1941-1955 (2006)

24. Liu, Wang-jin: Fuzzy proximity spaces redefined. Fuzzy Sets Syst. 15, 241-248 (1985)

25. Artico, G., Moresco, R.: Fuzzy proximities and totally bounded fuzzy uniformities. J. Math. Anal. Appl. 99, 320-337 (1984)

26. Lowen, R.: Fuzzy topological spaces and fuzzy compactness. J. Math. Anal. Appl. 56, 621-633 (1976)

27. El-Dardery, M., Ramadan, A.A., Kim, Y.C.: L-fuzzy topogenous orders and L-fuzzy topologies. J. Intell. Fuzzy Syst. 24, 685-691 (2013)

28. Šostak, A.: On a fuzzy topological structure. Suppl. Rend. Circ. Mat. Palermo Ser. II(11), 89-103 (1985)

29. Ramadan, A.A.: Smooth topological spaces. Fuzzy Sets Syst. 48, 371-375 (1992)

30. Kim, Y.C., Min, K.C.: L-fuzzy proximities and L-fuzzy topologies. Inf. Sci. 173, 93-113 (2005)

31. Čimoka, D., Šostak, A.P.: L-fuzzy syntopogenous structures, part I: fundamentals and application to L-fuzzy topologies, L-fuzzy proximities and L-fuzzy uniformities. Fuzzy Sets Syst. 232, 74-97 (2013)

32. Kim, Y.C., Oh, J.-M.: Alexandrov L-fuzzy pre-proximities. Mathematics 7(1), 1-15 (2019)

33. Ramadan, A.A., Elkordy, E.H., Kim, Y.C.: Perfect L-fuzzy topogenous spaces, L-fuzzy quasi-proximities and L-fuzzy quasi-uniform spaces. J. Int. Fuzzy Syst. 28, 2591-2604 (2015)

34. Ramadan, A.A., Usama, M.A., Reham, M.A.: On L-fuzzy pre-proximities and L-fuzzy interior operators. Ann. Fuzzy Math. Inf. 17(2), 191-204 (2019)

35. Pawlak, Z:: Rough sets. Int. J. Comput. Inf. Sci. 11, 341-356 (1982)

36. Wang, C.Y.: Topological characterizations of generalized fuzzy rough sets. Fuzzy Sets Syst. 312, 109-125 (2017)

37. Zhang, Li., Zhan, J.: Covering-based generalized IF rough sets with applications to multi-attribute decision-making. Inf. Sci. 478, 275-302 (2019)

38. Hájek, P.: Metamathematices of Fuzzy Logic. Kluwer Academic Publishers, Dordrecht (1998)

39. Oh, J.-M., Kim, Y.C.: L-Fuzzy closure operators and L-fuzzy cotopologies. J. Math. Comput. Sci. 9(2), 131-145 (2019)

40. Adámek, J., Herrlich, H., Strecker, G.E.: Abstract and Concrete Categories. Wiley, New York (1990)

\section{Publisher's note}

Springer Nature remains neutral with regard to jurisdictional claims in published maps and institutional affiliations.

\section{Submit your manuscript to a SpringerOpen ${ }^{\circ}$ journal and benefit from:}

- Convenient online submission

- Rigorous peer review

- Open access: articles freely available online

- High visibility within the field

Retaining the copyright to your article

Submit your next manuscript at $\boldsymbol{\Delta}$ springeropen.com 